\title{
Release of cartilage oligomeric matrix protein (COMP) into joint fluid after knee injury and in osteoarthritis
}

\author{
L Stefan Lohmander, Tore Saxne, Dick K Heinegård
}

\begin{abstract}
Objective-The release of fragments of cartilage oligomeric matrix protein (COMP) and aggrecan into knee joint fluid and serum after joint injury and in post-traumatic and primary osteoarthritis was monitored in a cross-sectional study.
\end{abstract}

Methods-Samples of joint fluid and serum were obtained from healthy volunteers and from patients with arthroscopically verified injury to anterior cruciate ligament and or meniscus of the knee at different times after injury and with different degrees of post-traumatic osteoarthritis. Samples were also obtained from patients with primary osteoarthritis. Fragments of COMP were quantified in joint fluid and in serum by enzyme-linked immunoassay. Fragments of aggrecan were quantified in joint fluid by enzymelinked immunoassay.

Results-Concentrations of COMP and aggrecan fragments in joint fluid in the study groups were increased over the reference levels of 47 (range 10-109) and 34 (range 6-59) $\mu \mathrm{g} / \mathrm{mL}$, respectively, in the volunteers with healthy knees. The ratios (w/w) of aggrecan to COMP fragments in joint fluid were also increased in all study groups over that in the reference group. Average concentrations of COMP in joint fluid were high shortly after injury and decreased with time but remained increased over reference levels for many years. Joint fluid COMP concentrations were also increased in early stage post-traumatic and primary osteoarthritis, but not in advanced osteoarthritis.

Conclusion-Increased amounts of aggrecan and COMP fragments are released into joint fluid after joint injury and in early stage osteoarthritis. The ratios of aggrecan to COMP in joint fluid vary with time after injury and with osteoarthritis disease stage. Although both molecules are sensitive to the agents active in matrix breakdown in joint disease, differences may thus exist in the release mechanisms and attempted repair. The concentrations of the individual markers in body fluids and their ratios may serve to monitor treatment efficacy, disease progression and repair in osteoarthritis and other joint diseases.

(Ann Rheum Dis 1994; 53: 8-13)

During turnover of cartilage matrix in normal and diseased joints, fragments of matrix molecules and other markers of cartilage metabolism are released into the synovial fluid. ${ }^{1-3}$ Injuries to the ligaments and menisci of the knee are associated with an increased risk for developing post-traumatic osteoarthritis and cause an increased release of fragments of joint cartilage proteoglycan (aggrecan) into joint fluid..$^{4-5}$ This increased release is maintained for many years after injury, perhaps as a result of changed loading patterns in the joint and resulting changes in the metabolism of the chondrocytes. A lowgrade, chronic synovitis may additionally contribute to these changes. Concurrently, dramatic increases are observed in the synovial fluid concentrations of the metalloproteinases stromelysin and collagenase and tissue inhibitor of metalloproteinases (TIMP). ${ }^{6-8}$ Further investigations on these and other markers of cartilage matrix metabolism in osteoarthritis and other joint diseases may improve our understanding of the disease mechanisms at the tissue and cell level and could also provide markers for monitoring disease activity, progress and therapy. ${ }^{9-13}$

Cartilage oligomeric matrix protein (COMP) is a cartilage-specific protein with a structural resemblance to thrombospondin but of unknown function. ${ }^{14}$ It consists of five subunits, each with an apparent $M_{r}$ of about $100 \mathrm{kda}^{15-16}$ The increased release of fragments of this protein into joint fluid and serum in rheumatoid arthritis, reactive arthritis and in primary osteoarthritis has recently been reported. ${ }^{12}{ }^{17-18}$ We now show in a crosssectional study that high concentrations of COMP are found in synovial fluid in connection with knee trauma. Further, elevated concentrations of this cartilage matrix component can be detected in joint fluid for many years after injury and during the development of both post traumatic and primary osteoarthritis.
S-22185 Lund,

Accepted for publication 28 January 1993 


\section{Patients and methods}

\section{PATIENTS AND DIAGNOSTIC CRITERIA}

Knee joint fluid samples were obtained from patients (one sample from each patient) at the time of admission to the emergency room, or at arthroscopy, at different times after knee injury and the beginning of knee symptoms. Patients and volunteers were assigned to the study groups defined below and in the table. All patient- and volunteer-related procedures were approved by the ethics committee of the Medical Faculty at Lund University.

The study group included: 6 healthy athlete volunteers (ATH) who had no previous history or clinical signs of joint disease or joint injury. Samples were obtained at least one day after physical training, 14 patients with no recent trauma but who experienced chronic knee-pain and in whom arthroscopy, radiology and blood chemistry had failed to reveal any pathology related to the knee-pain (NOR); 270 patients with knee trauma and tear of the anterior cruciate ligament, isolated or combined with tears of the medial or lateral meniscus (ACL). In addition some of these patients had collateral ligament tears; 63 patients with knee trauma and isolated tear of the medial or lateral meniscus (MEN). The diagnosis was confirmed by arthroscopy in all cases in groups NOR, ACL and MEN; 29 patients with no history of previous knee trauma or other knee disease but with arthroscopic and or radiographic evidence of joint cartilage damage and osteoarthritis (POA).

The condition of the knee joint was graded on an arbitrary scale (OA score) from 1 to 10 , based on a combination of findings at arthroscopy and or radiography ${ }^{19} ; 1$ : normal joint cartilage by arthroscopy; 2 : superficial fibrillations limited to one compartment; 3 : superficial fibrillations in more than one compartment; 4 : deep cartilage clefts limited to one compartment; 5: deep cartilage clefts in more than one compartment; 6 : subchondral sclerosis and minimal joint space decrease on standing $x$ ray films; $7:>50$ but $<100 \%$ decrease of joint space in at least one compartment; 8: $100 \%$ reduction of joint space in at least one compartment; 9: bony erosion $<5 \mathrm{~mm} ; 10$ : bony erosion $>5 \mathrm{~mm}$. Patients with OA scores of 1 to 5 thus all showed normal standing $x$ rays for subchondral sclerosis and joint space. Scores 6-10 represents increasing degrees of radiological changes on standing $x$ ray films indicative of osteoarthritis. $^{20}$

The diagnosis, sex, age at sampling, date of injury, date of sampling, volume of joint fluid

Patient data for diagnostic groups. Values are given as average (SD) median.

\begin{tabular}{|c|c|c|c|c|c|c|}
\hline Group $^{I}$ & $N$ & $A g e^{2}$ & $\operatorname{Sex}^{3}$ & Time $e^{4}$ & $O A^{5}$ & $\mathrm{Vol}^{6}$ \\
\hline ATH & 6 & $30(9) 28$ & 100 & - & - & $3(2) 3$ \\
\hline NOR & 14 & 28 (12) 25 & 64 & $55(47) 40$ & 1 & 2 (1) 1 \\
\hline $\mathrm{ACL}$ & 270 & 28 (10) 25 & 68 & $103(184) 22$ & $2(1) 1$ & $14(19) 6$ \\
\hline MEN & 63 & 40 (12) 39 & 79 & $176(310) 52$ & 2 (2) 2 & $12(19) 4$ \\
\hline POA & 29 & $55(20) 59$ & 69 & $170(154) 13$ & $6(2) 6$ & $29(34) 20$ \\
\hline
\end{tabular}

'See Patients and methods for diagnostic criteria.

${ }^{2}$ Age at sampling

${ }^{3}$ Percent male patients.

${ }^{4}$ Time after injury or duration of complaint (weeks).

${ }^{5} \mathrm{OA}$ score, see Patients and methods for definition.

${ }^{6}$ Volume of joint fluid aspirated $(\mathrm{mL})$. aspirated and $\mathrm{OA}$ score were registered together with joint fluid assay data (table). To study the relationship between time after joint injury and joint fluid marker concentrations, patients were sorted according to time after injury and divided into subgroups so that each consecutive 'time window' contained some 25 to 35 values. No patient included in the study was treated with intra-articular glucocorticoids or was on chronic treatment with non-steroidal anti-inflammatory drugs or had been treated by surgical reconstruction at the time of sampling.

\section{ASPIRATION OF JOINT FLUIDS}

Joint fluid samples were aspirated by a lateral parapatellar approach (with skin anaesthesia in volunteers), centrifuged at $3000 \times \mathrm{g}$ and aliquots of the supernatants were frozen and stored at $-70^{\circ} \mathrm{C}$ within a few hours of collection. Blood samples were drawn at the same time, serum separated within a few hours and frozen as above.

ASSAY OF AGGRECAN FRAGMENTS AND COMP Aggrecan fragments containing the chondroitin sulfate-binding domain were quantified in joint fluid by enzyme-linked immunoassay. ${ }^{9}$ Results are expressed as $\mu \mathrm{g}$ of intact human joint cartilage aggrecan per $\mathrm{mL}$ of joint fluid. COMP fragments in joint fluid or serum were quantified by enzyme-linked immunoassay. ${ }^{10}$ Results are expressed as $\mu \mathrm{g}$ of intact human COMP per $\mathrm{mL}$ of serum or joint fluid.

\section{STATISTICAL METHODS}

A non-parametric method (Mann-Whitney) was used to calculate statistical differences between groups. A probability value of less than 0.05 was considered to signify a difference between groups. Any differences between groups, increases over reference levels or correlations described as such in this paper are statistically significant at this level or better.

\section{Results}

The average concentration of COMP fragments in joint fluid in the reference group of 6 athletes with healthy knees (ATH) was 47 $\mu \mathrm{g} / \mathrm{mL}$ (range 10-109). Average concentrations of COMP in joint fluid in the groups with cruciate ligament injury, meniscus injury, primary osteoarthritis or with knee pain but no diagnosed knee pathology were all increased over the reference group (fig 1). Average concentrations of aggrecan fragments in joint fluid in the same four groups were likewise increased over the reference group (data not shown). The average ratio (w/w) of aggrecan to COMP in joint fluid in the reference group was $0 \cdot 8$ (range $0 \cdot 4-1 \cdot 3$ ), while the average ratios for the other groups ranged between $1 \cdot 1$ and $1 \cdot 4$ (fig 2). The differences in aggrecan to COMP ratios only reached statistical significance when comparing the cruciate ligament injury group and the reference group. 
COMP in synovial fluid

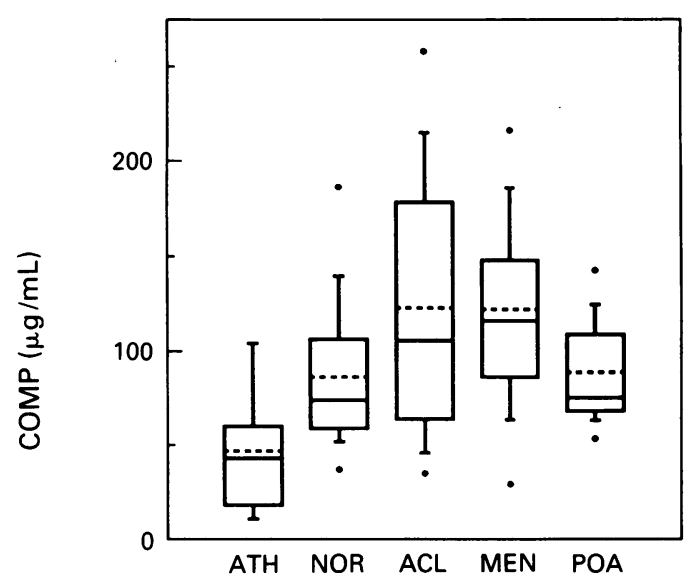

Figure 1 Concentrations of COMP fragments in joint fluid in the five study groups. Results are presented as box plots where the dividing line represents the median; the box contains 25th to 75th percentiles, whiskers contain 10th to 90th percentiles and dots contain 5th to 95th percentiles. $A T H=$ athletes with healthy knees; NOR = knee pain but normal arthroscopy; $A C L=$ anterior cruciate ligament injury with or without meniscus injury; $M E N=$ isolated meniscus injury; $P O A$ = primary osteoarthritis. See table for demographic data on study groups.

Ratio Agn/COMP in synovial fluid

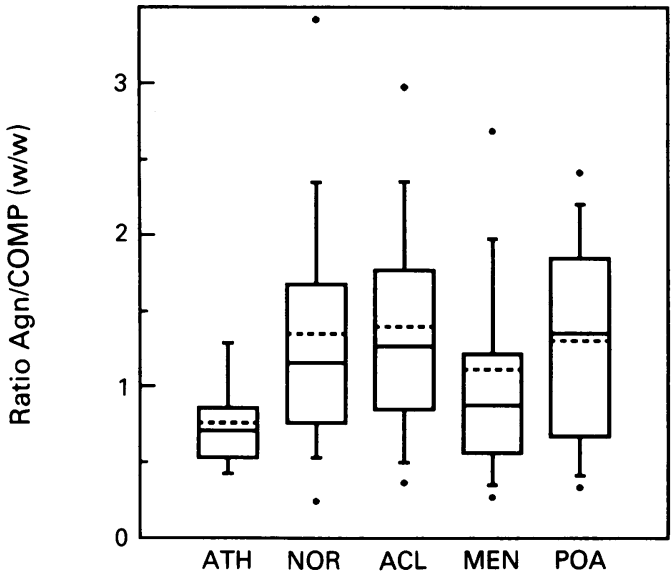

Figure 2 Ratios between aggrecan and COMP fragments (weight/weight) in joint fluid in the five study groups. See fig 1 for legend.

The serum concentration of COMP was also measured in a randomly selected subgroup $(\mathrm{N}=23)$ of the cruciate ligament injury patients (ACL). The average concentration in serum in this group was $9.6 \mu \mathrm{g} / \mathrm{mL}$, compared with an average synovial fluid concentration of $81.5 \mu \mathrm{g} / \mathrm{mL}$ in the same 23 patients. The serum concentration of COMP in this group did not differ from that of healthy blood donors. ${ }^{17}$ However, the serum concentration of COMP was positively correlated to the synovial fluid concentration $(r=0.52$, data not shown).

The knee injury groups ACL and MEN of this cross-sectional study both contained patients with different times between injury and sampling and also with different degrees of cartilage changes in their joints (table). We therefore analysed the relationship of these variables to joint fluid COMP and aggrecan concentrations in the combined injury groups

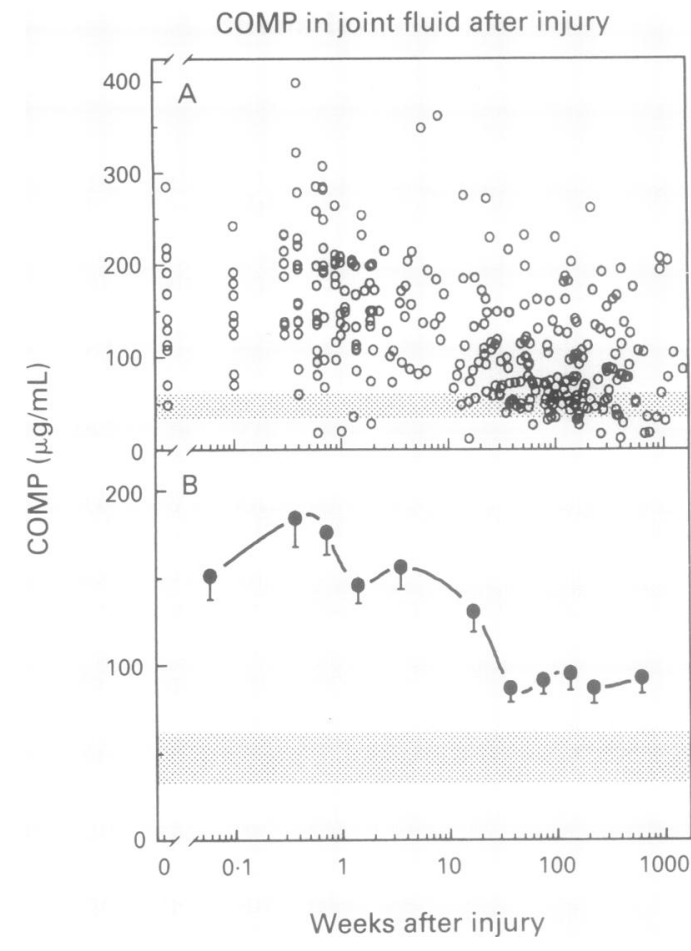

Figure 3 COMP fragment concentrations in joint fluid in 333 patients with knee injury (groups $A C L$ and MEN) in relation to time after injury. Patients were sorted according to time after injury and then subdivided so that each consecutive group contained some 25-35 patients each. (A) Scatter diagram for all individual values represented in panel $B$, with COMP concentration values related to time after injury; (B) Symbols represent average value for each 'time window', bars represent $\pm S E$. Shaded zone represents average $\pm S E$ for reference group $(A T H)$. Line was fitted by a cubic spline function. Please note different scale of $Y$-axis in panels $A$ and $B$.

of 333 samples (ACL and MEN). Average concentrations of both COMP and aggrecan fragments in joint fluid showed very high levels within hours after injury, with a more than four-fold increase over reference levels for both types of matrix molecule fragments 3-5 days after the trauma (fig 3), data shown only for COMP). At 6-12 months after the trauma, an average plateau level of about twice the reference concentration was reached and remained higher than the reference level for almost 20 years after injury. The range of values remained high at all time points.

Concentrations of COMP were positively correlated to the levels of aggrecan fragments in the injury groups $(r=0.56$, data not shown). In spite of this general correlation between the release of aggrecan and COMP fragments into joint fluid, the ratios (w/w) between the two components varied at different times after injury with a peak average value of about 1.8 at about five days and a gradual decrease towards one over time (fig 4). Later, ratios in the individual patients varied from less than one to about three. The average ratios for the time-related subgroups were higher than the average reference level at all times except for the first and last time points. Due to the limited number of patients with meniscus injury only, no comparison between the relative influence of cruciate ligament and meniscus injury on COMP release into joint fluid was made. There was no significant influence of age at 
Ratio of Agn/COMP in joint fluid after injury

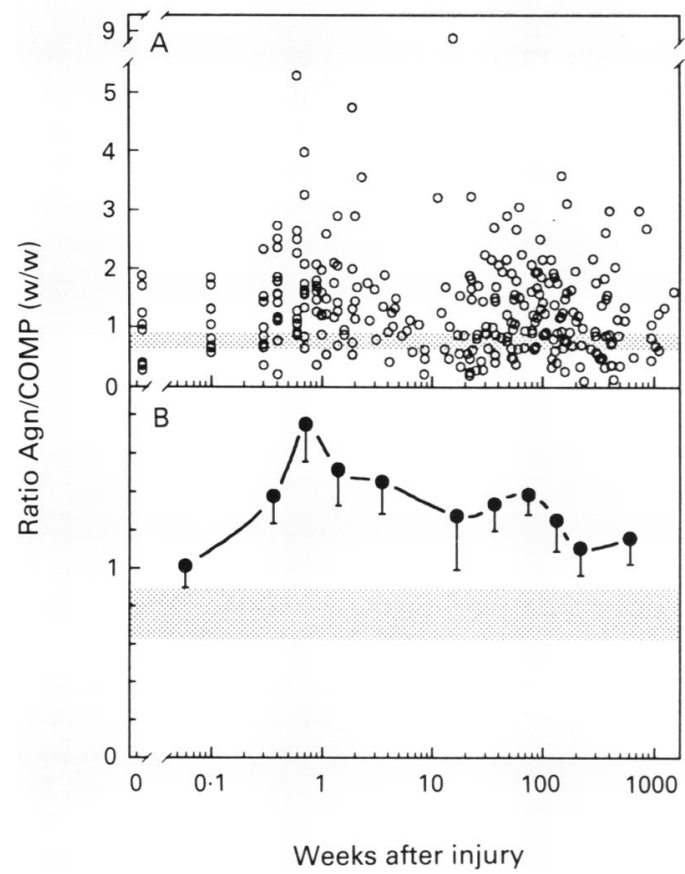

Figure 4 Ratios between concentrations of aggrecan (Agn) and COMP fragments in joint fluid in 333 patients with knee injury (groups $A C L$ and $M E N$ ) in relation to time after injury. See fig 3 for legend.

COMP in knee joint fluid $v$. OA score

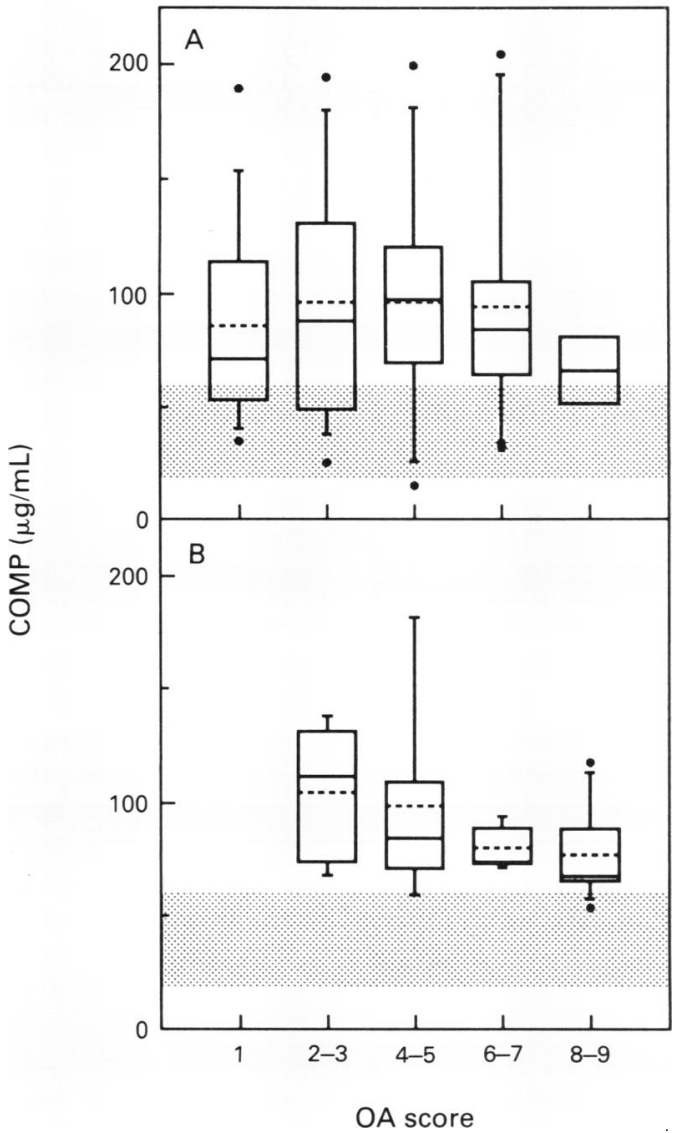

Figure 5 Concentrations of COMP fragments in joint fluid in $(A) 177$ patients with different stages of posttraumatic osteoarthritis (study groups ACL and MEN) or (B) 29 patients with different stages of primary osteoarthritis (study group POA). Patients with injury within six months of sampling were excluded. Shaded zone represents 25th to 75 th percentiles of reference group (ATH). See fig 1 for explanation of box plots. sampling or patient gender on COMP concentrations in joint fluid, once data were adjusted for differences in diagnosis, time after injury, etc.

The injury groups and the primary osteoarthritis groups both contained patients with joints at varying stages of cartilage damage. After excluding patients who were sampled within six months of injury (fig 3 ), the remaining 177 patients with injury to cruciate ligament and or meniscus were therefore stratified according to the OA score at sampling (see patients and methods). All 29 patients in the primary osteoarthritis group were stratified similarly. Concentrations of COMP fragments in joint fluid from injured joints were increased over the reference group in arthroscopically normal joints and in joints with all stages of osteoarthritis (OA scores 2-8) (fig $5 \mathrm{~A}$ ). The ratio of aggrecan to COMP in joint fluid was increased over the reference group only in arthroscopically normal joints (OA score $=1$ ), (fig $6 \mathrm{~A}$ ). For the limited number of joint fluid samples from patients with primary osteoarthritis, COMP concentrations were increased at OA scores 2-3 and 6-9, but not at scores $4-5$, perhaps due to great

Ratio of Agn/COMP in knee joint fluid v. OA score

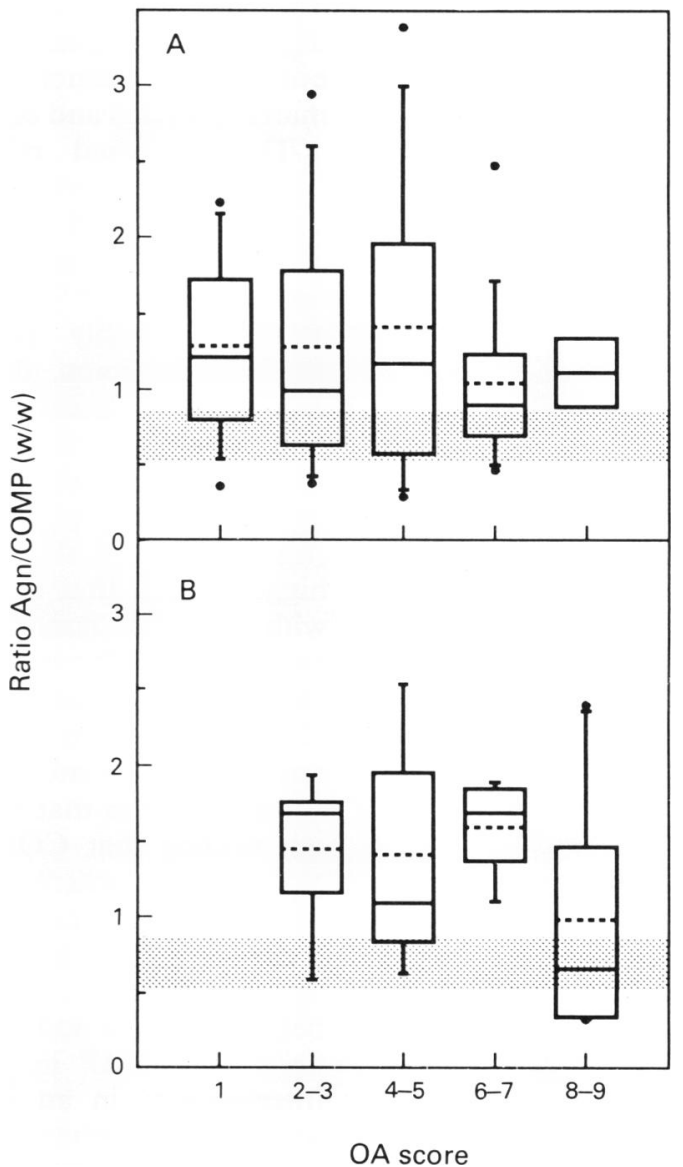

Figure 6 Ratios of aggrecan (Agn) to COMP fragment concentrations (w/w) in joint fluid in (A) 177 patients with different stages of post-traumatic osteoarthritis (study groups $A C L$ and $M E N$ ) or (B) 29 patients with different stages of primary osteoarthritis (study group POA). Patients with injury within six months of sampling were excluded. Shaded zone represents 25th to 75 th percentiles of reference group (ATH). See fig 1 for explanation of box plots. 
variability in this group (fig 5B). The ratio of aggrecan to COMP in primary osteoarthritis was increased over the reference value at $\mathrm{OA}$ scores 2-7 (fig 6B).

In this study we have chosen to present the information on release of cartilage matrix macromolecules into synovial fluid as concentration values. We have also evaluated the results expressed as total amounts (concentration $\times$ volume) of aggrecan or COMP fragments aspirated from each joint. The conclusions reached in this cross-sectional study were similar when the results were expressed as the mass of marker aspirated per joint. However, in agreement with previous studies, ${ }^{6} 1021$ we found a larger variability in values based on total mass than on concentration, due to the uncertainty involved in the measurement of total joint fluid volume.

\section{Discussion}

Earlier work on cartilage matrix turnover in normal and diseased cartilage and the ensuing release of metabolic products into body fluids, has mainly focused on the quantitative aspects of the release of aggrecan degradation products. With rapidly increasing knowledge of the structure, function and molecular biology of the different joint cartilage matrix molecules it is now becoming possible to monitor the turnover rates and also elucidate the degradation mechanisms not only of aggrecan but also of minor cartilage proteoglycans, matrix proteins and collagens. ${ }^{11} 1317$ 22-24

The increased release of fragments of cartilage oligomeric matrix protein, COMP, into synovial fluid and serum in inflammatory arthritis and primary osteoarthritis has been recently shown. ${ }^{12-18}$ The increased release of another possibly related cartilage matrix protein in joint disease has also been reported. ${ }^{25-26}$ Extending these reports, we now find increased synovial fluid concentrations of COMP after knee injury and in both posttraumatic and primary knee osteoarthritis (fig 1). We also show that COMP levels are highest early after injury, gradually decrease with time, but remain increased over healthy control levels for up to 20 years after injury (fig 3). This agrees with the observed higher COMP levels in acute stages of reactive arthritis than in later, chronic stages. ${ }^{12}$ Further agreement with that report is demonstrated in our finding that COMP levels in joint fluid change less markedly than do aggrecan levels, resulting in higher ratios of aggrecan to COMP levels early after injury than late after injury (fig 4). We also find a positive correlation between serum and synovial fluid concentrations of COMP in post-traumatic patients. Interestingly, in an investigation of patients with rheumatoid arthritis or reactive arthritis, a positive correlation between the marker in serum and synovial fluid was found only in monoarthrodial reactive arthritis. ${ }^{17}$

In spite of the significant increases in the average concentrations in joint fluid of both aggrecan and COMP fragment concentrations at later times after injury (fig $3 \mathrm{~A}$ ), it is also clear that many patients with knee injury after one to two years show concentrations for these markers which fall well within the normal range (fig 3B). ${ }^{4-8}$ Longitudinal studies on patient groups such as these will be required to determine if marker concentrations in this chronic phase of joint injury are predictive for later development of post-traumatic osteoarthritis. It is in this context of interest that elevated concentrations of aggrecan fragments in synovial fluid in newly diagnosed rheumatoid arthritis have been shown to be predictive for a high degree of joint destruction later in rheumatoid disease development. ${ }^{27}$

In the present study, concentrations of COMP in post-traumatic and primary osteoarthritis were increased over reference levels in the early stages of osteoarthritis development, but not later (fig 5). Again, release of aggrecan fragments was more pronounced than release of COMP fragments, which resulted in increased ratios of aggrecan to COMP in joint fluid early, but not late, in the disease (fig 6). This would concur with the findings in patients with inflammatory joint disease $^{1028}$ and in experimental and human osteoarthritis. ${ }^{19} 29$

The increase in COMP concentration and aggrecan to COMP ratio in joint fluid in the study group (NOR) with knee pain but with normal findings at arthroscopy, radiological and laboratory examination, suggests that this group may have contained some patients with as yet undiagnosed joint disease or injury.

The increased release of aggrecan, COMP and other cartilage matrix molecule fragments into joint fluid after injury is presumably due to both an increase in the rate of net matrix degradation and an increase in turnover rate due to repair attempts by the chondrocytes. In end-stage disease, cartilage matrix is destroyed as a result of net degradation and concomitant failure of repair. The increased release of both aggrecan and COMP fragments early and late after joint injury suggests that proteinases active in the affected cartilage matrix have the capability not only to degrade aggrecan but also other molecules of the matrix such as COMP. The reasons why there would seem to be a differential release of the various molecules of the cartilage matrix in response to injury and disease are, however, not clear. Perhaps a differing sensitivity to the agents promoting the release is one explanation, and differences in the concentrations of molecules between matrix compartments another. Different binding mechanisms to other components of the matrix may also contribute.

The structures of the COMP and aggrecan fragments released to joint fluid were not examined in the present study. However, it was previously shown that in rheumatoid joint fluids the major immunodetected molecular fragments of COMP had an apparent mass of $62 \mathrm{kDa}$, considerably smaller than the intact subunit protein. ${ }^{17}$ Fragments of aggrecan released to joint fluid after knee injury, and in osteoarthritis and inflammatory joint diseases have earlier been characterised in some detail. ${ }^{11}{ }^{13}$ They consist mainly of large 
fragments of the chondroitin sulfate-bearing domain, corresponding to core protein sizes of about 200, 170 and $135 \mathrm{kDa}$, respectively. They appear to result from a predominant proteolytic cleavage of the interglobular domain between the residues glutamate 373 and alanine 374 .

Identification of the cleavage sites in these and other molecular fragments of cartilage matrix may help to identify the active agents in cartilage matrix degradation and thus facilitate the development of therapies to prevent cartilage destruction in joint disease. In addition, the concentrations of the individual markers in body fluids and their ratios may serve to monitor treatment efficacy, disease progression and repair.

This study was supported by The Swedish Medical Research This study was supported by The Swedish Medical Research i Lund Fonder, Riksförbundet mot Reumatism, Konung Gustaf i Lund Fonder, Riksförbundet mot Reumatism, Konung Gustaf
V:s 80-årsfond, Crafoords Stiftelse, Axel and Margareth Ax:son V:s 80-årsfond, Crafoords Stiftelse, Axel and Margareth Ax:son
Johnsons Stiftelse, Greta and Johan Kocks Stiftelser, Nanna Johnsons Stiftelse, Greta and Johan Kocks Stiftelser, Nanna
Svartz fond, Thelma Zoegas Stiftelse, Alfred Österlunds Svartz fond, Thelma Zoegas Stiftelse, Alfred Österlunds
Stiftelse, CIBA-Geigy Corporation, Merck Research Laboratories and Procordias Forskningsstiftelse.

1 Heinegård D, Saxne T. Molecular markers of processes in cartilage in joint diseases. Brf Rheumatol 1991; 30 (suppl 1): $21-24$.

2 Lohmander L S, Lark M W, Dahlberg L, Walakovits L A, Roos H. Cartilage matrix metabolism in osteoarthritis: Markers in synovial fluid, serum and urine. Clin Biochem Markers in synovial

3 Thonar E J-M A, Manicourt D H, Williams J M, et al. Serum keratan sulfate. A measure of cartilage proteoglycan metabolism. In: Kuettner K, Schleyerbach R, Peyron J, Hascall V C, eds. Articular cartilage and osteoarthritis. New York: Raven Press, 1992: 429-45.

4 Lohmander L S, Dahlberg L, Ryd L, Heinegård D. Increased levels of proteoglycan fragments in knee joint fluid after injury. Arthritis Rheum 1989; 32: 1434-42.

5 Lohmander L S. Markers of cartilage metabolism in arthrosis. A review. Acta Orthop Scand 1991; 62: 623-32.

6 Lohmander L S, Hoerrner L A, Lark M W. Metalloproteinases, tissue inhibitor and proteoglycan fragments in knee synovial fluid in human osteoarthritis. Arthritis Rheum 1993; 36: 181-9.

7 Lohmander L S, Hoermer L A, Dahlberg L, Roos H, Björnsson $S$, Lark $M$ W. Stromelysin, tissue inhibitor and Björnsson $S$, Lark $M$ W. Stromelysin, tissue inhibitor and
proteoglycan fragments in human knee ioint fluid after proteoglycan fragments in human kn

8 Lohmander L S, Hoerner L A, Dahlberg L, Roos H, Lark $M \mathrm{~W}$. Temporal patterns of stromelysin, tissue inhibitor and proteoglycan fragments in synovial fluid after injury to knee cruciate ligament or meniscus. $\mathcal{F}$ Orthop Res 1993 (in press).

9 Saxne T, Heinegård D, Wollheim F A. Therapeutic effects on cartilage metabolism in arthritis as measured by release of proteoglycan structures into the synovial fluid. Ann Rheum Dis 1986; 45: 491-7.

10 Saxne $T$, Heinegård $D$. Synovial fluid analysis of two groups of proteoglycan epitopes distinguishes early and late of proteoglycan epitopes distinguishes early and
cartilage lesions. Arthritis Rheum 1992; 35: 385-90.
11 Sandy J D, Flannery C R, Neame P J, Lohmander L S. The structure of aggrecan fragments in human synovial fluid. structure of aggrecan fragments in human synovial fluid. Evidence for the involvement in osteoarthritis of a novel
proteinase which cleaves the glu 373 -ala 374 bond of the proteinase which cleaves the glu 373-ala 374 bond of the

12 Saxne T, Glennås A, Kvien T K, Melby K, Heinegård D. Release of cartilage macromolecules into synovial fluid in acute and prolonged phases of reactive arthritis. Arthritis Rheum 1993; 36: 20-25.

13 Lohmander L S, Neame P J, Sandy J D. The structure of aggrecan fragments in human synovial fluid: Evidence that aggrecanase mediates cartilage degradation in inflammatory joint disease, joint injury and osteoarthritis. Arthritis Rheum 1993; 36: 1814-22.

14 Oldberg $\AA$, Antonsson $\mathrm{P}$, Lindblom K, Heinegård D. COMP (Cartilage Oligomeric Matrix Protein) is structurally related to the Thrombospondins. $\mathcal{F}$ Biol Chem 1992; 267: 22346-50

15 Hedbom E, Antonsson P, Hjerpe A, et al. Cartilage matrix proteins-an acidic oligomeric protein (COMP) detected only in cartilage. $\mathcal{F}$ Biol Chem 1992; 267: 6132-6.

16 Mörgelin $M$, Heinegård $D$, Engel J, Paulsson M. Electron microscopy of native cartilage oligomeric matrix protein purified from the Swarm rat chondrosarcoma reveals a five-armed structure. F Biol Chem 1992; 267: 6137-141.

17 Saxne T, Heinegård D. Cartilage oligomeric matrix protein: a novel marker of cartilage turnover detectable in synovial fluid and blood. Br $¥$ Rheumatol 1992; 31: 583-91.

18 Forslind K, Eberhardt K, Jonsson A, Saxne T. Increased serum concentrations of cartilage oligomeric matrix protein. A prognostic marker in early rheumatoid protein. A prognostic marker in early
arthritis. Br $\mathcal{F}$ Rheumatol 1992; 31: 593-8.

19 Dahlberg L, Ryd L, Heinegård D, Lohmander L S Proteoglycan fragments in joint fluid-influence of arthrosis and inflammation. Acta Orthop Scand 1992; 63: 417-23.

20 Ahlbäck S. Osteoarthritis of the knee: a radiographic investigation. Acta Radiol (Stockholm) 1968; suppl 277: 7-72.

21 Geborek P, Saxne T, Heinegård D, Wollheim F A. Measurement of synovial fluid volume using albumin dilution upon intra-articular saline injection. $\mathcal{F}$ Rheumatol 1988; 15: 91-94.

22 Heinegård D, Oldberg $\AA$. Structure and biology of cartilage and bone matrix non-collagenous macromolecules. and bone matrix non-coll

23 Doege K J, Sasaki M, Kimura T, Yamada Y. Complete coding sequence and deduced primary structure of the human cartilage large aggregating proteoglycan, aggrecan. f Biol Chem 1991; 266: 894-902.

24 Dodge G R, Poole A R. Immunohistochemical detection and immunochemical analysis of Type II collagen degradation in human normal, rheumatoid, and osteoarthritic articular cartilages and in explants of bovine articular cartilage cultured with interleukin 1.7 Clin Invest 1989; 83: 647-61.

25 Fife $R$. Identification of cartilage matrix glycoprotein in synovial fluid in human osteoarthritis. Arthritis Rheum 1988; 31: 553-6.

26 Fife $\mathrm{R}$, Brandt $\mathrm{K} D$, Braunstein $\mathrm{E} M$, et al. The presence of cartilage matrix glycoprotein in serum as determined "early" osteoarthritis of the knee. $₹$ Lab Clin Med 1991; "early" oste

27 Saxne T, Wollheim F A, Pettersson H, Heinegård D. Proteoglycan concentration in synovial fluid: predictor of future cartilage destruction in rheumatoid arthritis? $B M \mathcal{F}$ 1987; 295: 1447-8.

28 Saxne T, Heinegård $D$, Wollheim $F$ A, Pettersson $H$. Difference in cartilage proteoglycan level in synovial fluid in early rheumatoid arthritis and reactive arthritis. Lancet 1985; 8447: 127-128.

29 Messner K, Gillquist J, Björnsson S, Lohmander L S. Proteoglycan fragments in rabbit joint fluid correlated to arthrosis stage. Acta Orthop Scand 1993; 64: 312-16. 